\title{
Multi-Attribute Group Decision Making Based on Multigranulation Probabilistic Models with Interval-Valued Neutrosophic Information
}

\author{
Chao Zhang ${ }^{1}$, Deyu Li ${ }^{1, *}$, Xiangping Kang ${ }^{2,3}$, Yudong Liang ${ }^{1}$, Said Broumi ${ }^{4}$ and \\ Arun Kumar Sangaiah ${ }^{5}$ \\ 1 Key Laboratory of Computational Intelligence and Chinese Information Processing of Ministry of Education, \\ School of Computer and Information Technology, Shanxi University, Taiyuan 030006, China; \\ czhang@sxu.edu.cn (C.Z.); liangyudong@sxu.edu.cn (Y.L.) \\ 2 Key Laboratory of Embedded System and Service Computing, Ministry of Education, \\ Department of Computer Science and Technology, Tongji University, Shanghai 201804, China; \\ tongji_kangxp@sina.com \\ 3 China National Tobacco Corporation, Shanxi, Taiyuan 030000, China \\ 4 Laboratory of Information Processing, Faculty of Science Ben M'Sik, University Hassan II, Sidi Othman, \\ Casablanca B.P 7955, Morocco; broumisaid78@gmail.com \\ 5 School of Computing Science and Engineering, Vellore Institute of Technology (VIT), Vellore 632014, India; \\ arunkumarsangaiah@gmail.com \\ * Correspondence: lidysxu@163.com
}

Received: 8 January 2020; Accepted: 4 February 2020; Published: 9 February 2020

check for updates

\begin{abstract}
In plenty of realistic situations, multi-attribute group decision-making (MAGDM) is ubiquitous and significant in daily activities of individuals and organizations. Among diverse tools for coping with MAGDM, granular computing-based approaches constitute a series of viable and efficient theories by means of multi-view problem solving strategies. In this paper, in order to handle MAGDM issues with interval-valued neutrosophic (IN) information, we adopt one of the granular computing ( $\mathrm{GrC}$ )-based approaches, known as multigranulation probabilistic models, to address IN MAGDM problems. More specifically, after revisiting the related fundamental knowledge, three types of IN multigranulation probabilistic models are designed at first. Then, some key properties of the developed theoretical models are explored. Afterwards, a MAGDM algorithm for merger and acquisition target selections (M\&A TSs) with IN information is summed up. Finally, a real-life case study together with several detailed discussions is investigated to present the validity of the developed models.
\end{abstract}

Keywords: multi-attribute group decision-making; granular computing; interval-valued neutrosophic information; multigranulation probabilistic models; merger and acquisition target selections

\section{Introduction}

\subsection{A Brief Review of MAGDM}

By applying decision-making issues with multiple attributes to the setting of group decision-making, multi-attribute group decision-making (MAGDM) generally provides consistent group preferences by analyzing various alternatives expressed by individual preferences [1]. To date, many granular computing ( $\mathrm{GrC}$ )-based approaches [2-6] have been utilized to solve numerous complicated MAGDM problems, which have started a new momentum of constant development of social economy. 
In the process of solving a typical MAGDM problem, it is recognized that three key challenges need to be managed reasonably, i.e., MAGDM information representation, MAGDM information fusion, MAGDM information analysis. Among the above-stated key challenges, how to express MAGDM information, especially for a complicated uncertain real-world scenario, as a standard decision matrix via alternatives and attributes is the first step to address MAGDM problems.

\subsection{A Brief Review of Interval-valued Neutrosophic Information}

In order to meet the demands of describing fuzzy and indeterminate information at the same time from nature and society, Smarandache $[7,8]$ founded the notion of neutrosophic sets (NSs), which can be regarded as many generalizations of extended fuzzy sets [9] and used in plenty of meaningful areas [10-12]. An NS contains three types of membership functions (the truth, indeterminacy and falsity ones), and all of them take values in $] 0^{-}, 1^{+}[$. In accordance with the mathematical formulation of NSs, using NSs directly to a range of realistic applications is relatively inconvenient because all membership functions are limited within $] 0^{-}, 1^{+}[$. Thus, it is necessary to update $] 0^{-}, 1^{+}[$by virtue of standard sets and logic. Following the above-stated research route, Wang et al. [13] put forward the concept of IN sets (INSs) from the viewpoint of definitions, operational laws, and others. For each membership function in INSs, all of them take values in the power set of $[0,1]$ instead of $] 0^{-}, 1^{+}[$. Thus, INSs can tackle the first key challenge of solving typical MAGDM problems well [14-22].

\subsection{A Brief Review of Multigranulation Probabilistic Models}

To handle MAGDM information fusion and analysis with IN information effectively, GrC-based approaches own unique superiorities in constructing problem addressing approaches via multi-view problem solving tactics $[23,24]$. During the past several years, taking full advantage of GrC-based approaches, many scholars and practitioners have obtained fruitful results in merging NSs with rough sets [25-32], formal concept analysis [33,34], three-way decisions [35-37], and others [38,39]. In the current article, we plan to propose a new IN MAGDM method via multigranulation probabilistic models, which can provide a risk-based information synthesis scheme with the capability of error tolerance in light of GrC-based approaches. In particular, the notion of multigranulation rough sets (MGRSs) [40-42] and probabilistic rough sets (PRSs) [43-45] is scheduled to establish multigranulation probabilistic models, and the merits of MGRSs and PRSs can be reflected in the process of MAGDM problem addressing.

\subsection{The Motivations of the Research}

MGRSs play a significant role in dealing with MAGDM problems in diverse backgrounds. One one hand, some scholars have made eminent contributions to the applications of MGRSs in MAGDM problems in recent years. For instance, Zhang et al. [46,47] developed various MGRSs in the context of hesitant fuzzy and interval-valued hesitant fuzzy sets for handling person-job fit and steam turbine fault diagnosis, respectively. Sun et al. [48,49] proposed several MGRSs with linguistic and heterogeneous preference information, then they further designed corresponding MAGDM approaches. Zhan et al. [50] and Zhang et al. [51] put forward two novel covering-based MGRSs with fuzzy and intuitionistic fuzzy information for addressing MAGDM problems. On the other hand, some scholars adopted PRSs to address MAGDM problems. For instance, Liang et al. [52-54] studied novel decision-theoretic rough sets in hesitant fuzzy, incomplete, and Pythagorean information systems. $\mathrm{Xu}$ and Guo [55] generalized MGRSs to double-quantitative and three-way decision frameworks. Zhang et al. [56,57] combined decision-theoretic rough sets with MGRSs in Pythagorean and hesitant fuzzy linguistic information systems. In this paper, we generalize MGRSs and PRSs to IN information and apply them to M\&A TSs. Specifically, the following motivations of utilizing MGRSs and PRSs in IN MAGDM problems can be summed up: 
1. In order to address the challenge of MAGDM information representation, INSs take advantages of NSs and interval-valued sets at the same time. Thus, INSs play a significant role in describing indeterminate and incomplete MAGDM information.

2. In order to address the challenge of MAGDM information fusion, MGRSs excel in accelerating the information fusion procedure by virtue of processing multiple binary or fuzzy relations in parallel. In addition, classical MGRSs offer decision makers both an optimistic information fusion rule and a pessimistic counterpart. In conclusion, MGRSs play a significant role in constructing reasonable MAGDM information fusion methods [46-51].

3. In order to address the challenge of MAGDM information analysis, starting from the probability theory and Bayesian procedures, PRSs own the capability of fault tolerance. Hence, PRSs play a significant role in coping with incorrect and noisy data and they can be seen as a useful tool for robust MAGDM information analysis [52-57].

\subsection{The Contributions of the Research}

In this work, we aim to utilize MGRSs and PRSs in solving complicated MAGDM issues with IN information. Specifically, several comprehensive risk-based models named IN multigranulation PRSs (MG-PRSs) over two universes are looked into. Then, we further present a MAGDM approach in the setting of M\&A TSs in light of the developed theoretical models that can avoid an impact of three above-mentioned challenges. Finally, a real-world example is employed to prove the validity of the established decision-making rule. In addition, it is noteworthy that plenty of interesting nonlinear modeling approaches have been proved to be successful in various applications [58-67]. For instance, Medina and Ojeda-Aciego [58] applied multi-adjoint frameworks to general $t$-concept lattice, and some other works on fuzzy formal contexts based on GrC-based approaches were explored in succession [64-67]. Takacs et al. [59] put forward a brand-new oft tissue model for constructing telesurgical robot systems. Gil et al. [60] studied a surrogate model based optimization of traffic lights cycles and green period ratios by means of microscopic simulation and fuzzy rule interpolation. Smarandache et al. [61] explored word-level sentiment similarities in the context of NSs, and some other meaningful works on word-level sentiment analysis were also investigated recently $[62,63,68,69]$.

Compared with existing popular nonlinear modeling approaches, the vital contributions of the work lie in the utilization of IN information and multigranulation probabilistic models. For one thing, the above-mentioned literature on nonlinear modeling approaches can not process various practical situations with indeterminate and incomplete information effectively, thus this work shows some merits in the representation of uncertain MAGDM information. For another, the majority of nonlinear modeling approaches can not fuse and analyze multi-source information with incorrect and noisy data reasonably, thus this work shows some merits in the fusion and analysis of MAGDM problems with IN information. Moreover, several specific key contributions of the work can be further concluded below:

1. Several new IN membership degrees are put forward to handle incorrect and noisy data via the capability of fault tolerance.

2. Three types of multigranulation probabilistic models are designed according to diverse risk attitudes of decision makers, i.e., the first one in light of optimistic rules, the second one in light of pessimistic rules, and the third one in light of adjustable rules.

3. On the basis of GrC-based methods, IN MG-PRSs over two universes can address a typical IN MAGDM issue from multiple views, and integrate different individual preferences by considering risk appetites and error tolerance.

\subsection{The Structure of the Research}

The rest of the work is arranged below. The next section intends to review several basic knowledge on INSs, MGRSs, and PRSs. Three types of theoretical models along with their key properties are explored in Section 3. In the next section, we develop an IN MAGDM approach via multigranulation 
probabilistic models in the context of M\&A TSs. In Section 5, a practical illustrative case study is explored to highlight the validity of the presented IN MAGDM rule. Finally, Section 6 contains several conclusive results and future study options.

\section{Preliminaries}

The current section plans to revisit various preliminary knowledge in terms of INSs, MGRSs, and PRSs in a brief outline.

\subsection{INSs}

The concept of INSs was put forward by Wang et al. [13] by updating the formulation of $] 0^{-}, 1^{+}[$ from the scope of membership functions in NSs. With this update, INSs are equipped with the capability of expressing indeterminate and incomplete information simultaneously.

Definition 1 ([13]). Suppose $U$ is an arbitrary universe of discourse. An INS E over $U$ is provided as the following mathematical expression:

$$
E=\left\{\left\langle x,\left[\mu_{E}^{L}(x), \mu_{E}^{U}(x)\right],\left[v_{E}^{L}(x), v_{E}^{U}(x)\right],\left[\omega_{E}^{L}(x), \omega_{E}^{U}(x)\right]\right\rangle \mid x \in U\right\},
$$

where $\mu, v, \omega: U \rightarrow$ int $[0,1]$ (int $[0,1]$ represents the set of all closed subintervals of $[0,1]$ ). Similar with NSs, there also exists the restriction of $0 \leq \mu_{E}^{U}(x)+v_{E}^{U}(x)+\omega_{E}^{U}(x) \leq 3$. Moreover, a set that includes all INSs over $U$ is further named $I N(U)$.

For an INS $E$, it is noticed that $E$ may degenerate into two special forms, i.e., an INS $E$ is named a full INS $U$ when $\left[\mu_{E}^{L}(x), \mu_{E}^{U}(x)\right]=[1,1],\left[v_{E}^{L}(x), v_{E}^{U}(x)\right]=[0,0]$ and $\left[\omega_{E}^{L}(x), \omega_{E}^{U}(x)\right]=[0,0]$; an INS $E$ is named an empty INS $\varnothing$ when $\left[\mu_{E}^{L}(x), \mu_{E}^{U}(x)\right]=[0,0],\left[v_{E}^{L}(x), v_{E}^{U}(x)\right]=[1,1]$ and $\left[\omega_{E}^{L}(x), \omega_{E}^{U}(x)\right]=[1,1]$.

In IN MAGDM information analysis, it is common to compare the magnitude of IN numbers, thus a frequently-used method was developed in [15].

Definition 2 ([15]). Suppose $x=\left\langle\left[\mu_{E}^{L}(x), \mu_{E}^{U}(x)\right],\left[v_{E}^{L}(x), v_{E}^{U}(x)\right],\left[\omega_{E}^{L}(x), \omega_{E}^{U}(x)\right]\right\rangle$ is an IN number. A score function with regard to $x$ is provided as the following mathematical expression:

$$
s(x)=\left[\mu_{E}^{L}(x)+1-v_{E}^{U}(x)+1-\omega_{E}^{U}(x), \mu_{E}^{U}(x)+1-v_{E}^{L}(x)+1-\omega_{E}^{L}(x)\right],
$$

for arbitrary two IN numbers $x$ and $y, x \leq y \Leftrightarrow s(x) \leq s(y)$ is evident.

It is noted that another significant issue in IN MAGDM information analysis is viable operational laws, which are used in constructing various IN MAGDM models. In what follows, we present several IN operational laws.

Definition $3([13,15,22])$. Suppose $E$ and $F$ are arbitrary two INSs over $U$, then some common IN operational laws are provided as the following mathematical expressions:

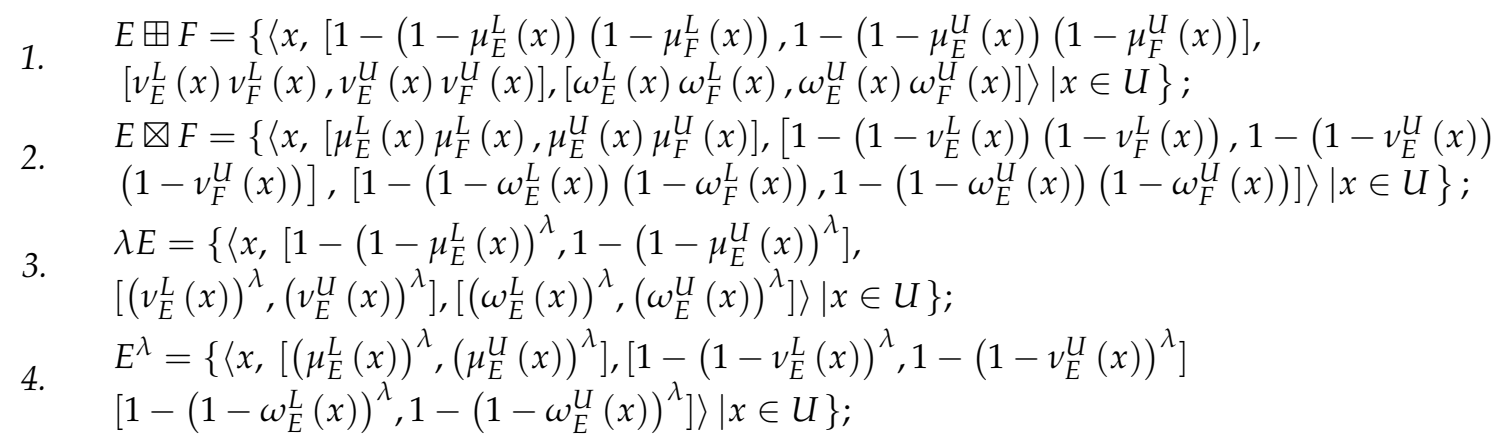


5. $\quad E \boxminus F=\left\{\left\langle x,\left[\frac{\mu_{E}^{L}(x)-\mu_{F}^{L}(x)}{1-\mu_{F}^{L}(x)}, \frac{\mu_{E}^{U}(x)-\mu_{F}^{U}(x)}{1-\mu_{F}^{U}(x)}\right],\left[\frac{v_{E}^{L}(x)}{v_{F}^{L}(x)}, \frac{v_{E}^{U}(x)}{v_{F}^{U}(x)}\right],\left[\frac{\omega_{E}^{L}(x)}{\omega_{F}^{L}(x)}, \frac{\omega_{E}^{U}(x)}{\omega_{F}^{U}(x)}\right]\right\rangle \mid x \in U\right\}$, if $E \supseteq F, v_{E}^{L}(x) \leq$ $\frac{v_{E}^{U}(x) \nu_{F}^{L}(x)}{v_{F}^{U}(x)}$ and $\omega_{E}^{L}(x) \leq \frac{\omega_{E}^{U}(x) \omega_{F}^{L}(x)}{\omega_{F}^{U}(x)}$;

6. $\quad E \boxminus F=\left\{\left\langle x,\left[\frac{\mu_{E}^{L}(x)}{\mu_{F}^{L}(x)}, \frac{\mu_{E}^{U}(x)}{\mu_{F}^{U}(x)}\right],\left[\frac{v_{E}^{L}(x)-\nu_{F}^{L}(x)}{1-v_{F}^{L}(x)}, \frac{v_{E}^{U}(x)-v_{F}^{U}(x)}{1-v_{F}^{U}(x)}\right],\left[\frac{\omega_{E}^{L}(x)-\omega_{F}^{L}(x)}{1-\omega_{F}^{L}(x)}, \frac{\omega_{E}^{U}(x)-\omega_{F}^{U}(x)}{1-\omega_{F}^{U}(x)}\right]\right\rangle \mid x \in U\right\}$, if $F \supseteq E$ and $\omega_{E}^{L}(x) \leq \frac{\omega_{E}^{U}(x) \omega_{F}^{L}(x)}{\omega_{F}^{U}(x)}$

7. $E^{c}=\left\{\left\langle x,\left[\omega_{E}^{L}(x), \omega_{E}^{U}(x)\right],\left[1-v_{E}^{U}(x), 1-v_{E}^{L}(x)\right],\left[\mu_{E}^{L}(x), \mu_{E}^{U}(x)\right]\right\rangle \mid x \in U\right\}$;

8. $E \cup F=\left\{\left\langle x,\left[\max \left(\mu_{E}^{L}(x), \mu_{F}^{L}(x)\right), \max \left(\mu_{E}^{U}(x), \mu_{F}^{U}(x)\right)\right],\left[\min \left(v_{E}^{L}(x), v_{F}^{L}(x)\right)\right.\right.\right.$, $\left.\left.\left.\min \left(v_{E}^{U}(x), v_{F}^{U}(x)\right)\right],\left[\min \left(\omega_{E}^{L}(x), \omega_{F}^{L}(x)\right), \min \left(\omega_{E}^{U}(x), \omega_{F}^{U}(x)\right)\right]\right\rangle \mid x \in U\right\} ;$

9. $E \cap F=\left\{\left\langle x,\left[\min \left(\mu_{E}^{L}(x), \mu_{F}^{L}(x)\right), \min \left(\mu_{E}^{U}(x), \mu_{F}^{U}(x)\right)\right],\left[\max \left(v_{E}^{L}(x), v_{F}^{L}(x)\right)\right.\right.\right.$, $\left.\left.\left.\max \left(v_{E}^{U}(x), v_{F}^{U}(x)\right)\right],\left[\max \left(\omega_{E}^{L}(x), \omega_{F}^{L}(x)\right), \max \left(\omega_{E}^{U}(x), \omega_{F}^{U}(x)\right)\right]\right\rangle \mid x \in U\right\} ;$

10. $E \subseteq F \Leftrightarrow \mu_{E}^{L}(x) \leq \mu_{F}^{L}(x), \mu_{E}^{U}(x) \leq \mu_{F}^{U}(x), v_{E}^{L}(x) \geq v_{F}^{L}(x), v_{E}^{U}(x) \geq v_{F}^{U}(x), \omega_{E}^{L}(x) \geq$ $\omega_{F}^{L}(x), \omega_{E}^{U}(x) \geq \omega_{F}^{U}(x)$

11. $E=F \Leftrightarrow E \subseteq F, E \supseteq F$.

\subsection{MGRSs}

As one of the most influential generalized rough set theories, the idea of MGRSs was initially established by Qian et al. [40-42] by means of parallel computational frameworks and risk-based information fusion strategies.

Definition 4 ([40,41]). Suppose $R_{1}, R_{2}, \ldots, R_{m}$ are $m$ crisp binary relations. For any $X \subseteq U$, the optimistic and pessimistic multigranulation approximations of $X$ are provided as the following mathematical expressions:

$$
\begin{aligned}
& \sum_{i=1}^{m} R_{i}^{O}(X)=\left\{[x]_{R_{1}} \subseteq X \vee[x]_{R_{1}} \subseteq X \vee \ldots \vee[x]_{R_{m}} \mid x \in U\right\} ; \\
& {\overline{\sum_{i=1}^{m} R_{i}}}^{O}(X)=\left(\sum_{i=1}^{m} R_{i}\left(X^{c}\right)\right)^{c} \text {; } \\
& \begin{array}{l}
\sum_{i=1}^{m} R_{i} \quad(X)=\left\{[x]_{R_{1}} \subseteq X \wedge[x]_{R_{1}} \subseteq X \wedge \ldots \wedge[x]_{R_{m}} \mid x \in U\right\} ; \\
{\overline{\sum_{i=1}^{m} R_{i}}}^{P}(X)=\left(\sum_{i=1}^{m} R_{i}^{P}\left(X^{c}\right)\right)^{c},
\end{array}
\end{aligned}
$$

the pair $\left(\sum_{i=1}^{m} R_{i}^{O}(X),{\overline{\sum_{i=1}^{m} R_{i}}}^{O}(X)\right)$ is named an optimistic MGRS with regard to $X$, whereas the pair $\left(\sum_{i=1}^{m} R_{i}^{P}(X), \overline{\sum_{i=1}^{m}} R_{i}(X)\right)$ is named a pessimistic MGRS with regard to $X$.

\section{3. $P R S S$}

Considering that the formulation of classical rough sets is fairly strict which may affect the application range of it, hence the concept of PRSs [43-45] was developed subsequently by means of the probabilistic measure theory. 
Definition 5 ([43]). Suppose $R$ is an equivalence relation over $U, P$ is the probabilistic measure, then $(U, R, P)$ is named a probabilistic approximation space. For any $0 \leq \beta<\alpha \leq 1$ and $X \subseteq U$, the lower and upper approximations of $X$ are provided as the following mathematical expressions:

$$
\begin{aligned}
& \underline{R}_{\alpha}(X)=\left\{P\left(X \mid[x]_{R}\right) \geq \alpha \mid x \in U\right\} ; \\
& \bar{R}_{\beta}(X)=\left\{P\left(X \mid[x]_{R}\right)>\beta \mid x \in U\right\},
\end{aligned}
$$

the pair $\left(\underline{R}_{\alpha}(X), \bar{R}_{\beta}(X)\right)$ is named a PRS of $X$ with regard to $(U, R, P)$.

\section{IN MG-PRSs over Two Universes}

In what follows, prior to the introduction of new theoretical models, we shall revisit the formulation of IN relations within the context of two universes [28] at first.

Definition 6 ([28]). Suppose $U$ and $V$ are two an arbitrary universes of discourse. An IN relation over two universes $R$ over $U \times V$ is provided as the following mathematical expression:

$$
R=\left\{\left\langle(x, y),\left[\mu_{R}^{L}(x, y), \mu_{R}^{U}(x, y)\right],\left[v_{R}^{L}(x, y), v_{R}^{U}(x, y)\right],\left[\omega_{R}^{L}(x, y), \omega_{R}^{U}(x, y)\right]\right\rangle \mid(x, y) \in U \times V\right\},
$$

where $\mu, v, \omega: U, V \rightarrow$ int $[0,1]$. Similar with INSs, there also exists the restriction of $0 \leq \mu_{R}^{U}(x, y)+$ $v_{R}^{U}(x, y)+\omega_{R}^{U}(x, y) \leq 3$. Moreover, a set that includes all IN relations over $U \times V$ is further named $\operatorname{INR}(U \times V)$.

\subsection{Optimistic IN MG-PRSs over Two Universes}

It is noted that the term "optimistic" is originated from the first paper of MGRSs [40]. Within the context of MGRSs, the notion of single and multiple IN inclusion degrees is scheduled to propose at first in the current section.

Definition 7. Suppose $R_{i}$ is an IN relation over two universes over $U \times V$. For any $E \in I N(V)$, $x \in U, y \in V$, the single IN membership degree of $x$ in $E$ in terms of $R_{i}$ is provided as the following mathematical expression:

$$
\eta_{E}^{R_{i}}(x)=\frac{\sum_{y \in V} R_{i}(x, y) E(y)}{\sum_{y \in V} R_{i}(x, y)}
$$

based on $\eta_{E}^{R_{i}}(x)$, the multiple IN membership degrees of $x$ in $E$ with regard to $R_{i}$ are provided as the following mathematical expressions:

$$
\begin{aligned}
& \Psi_{E}^{\sum_{i=1}^{m} R_{i}}(x)=\min _{i=1}^{m} \eta_{E}^{R_{i}}(x) ; \\
& \Omega_{E}^{\sum_{i=1}^{m} R_{i}}(x)=\max _{i=1}^{m} \eta_{E}^{R_{i}}(x),
\end{aligned}
$$

$\sum_{E}^{\sum_{i=1}^{m} R_{i}}(x)$ is named a minimal IN membership degree, whereas we call $\Omega_{E}^{\sum_{i=1}^{m} R_{i}}(x)$ a maximal IN membership degree.

In light of maximal IN membership degrees, optimistic multigranulation probabilistic models can be put forward conveniently. 
Definition 8. Suppose $R_{i}$ is an IN relation over two universes over $U \times V$. For any $E \in I N(V), x \in U$, $y \in V$, the two IN thresholds are represented by $\alpha$ and $\beta$ with $\alpha>\beta$, then the lower and upper approximations of $E$ in optimistic multigranulation probabilistic models are provided as the following mathematical expressions:

$$
\begin{aligned}
& {\overline{\sum_{i=1}^{m} R_{i}, \alpha}(E)=\left\{\Omega_{E}^{\sum_{i=1}^{m} R_{i}}(x) \geq \alpha \mid x \in U\right\} ;}^{\sum_{i=1}^{m} R_{i}, \beta}(E)=\left\{\Omega_{E}^{\sum_{i=1}^{m} R_{i}}(x)>\beta \mid x \in U\right\},
\end{aligned}
$$

the pair $\left(\sum_{i=1}^{m} R_{i}^{\Omega, \alpha}(E), \sum_{i=1}^{m} R_{i}, \beta(E)\right)$ is named an optimistic IN MG-PRS over two universes of E.

In what follows, some key properties of lower and upper approximations for optimistic multigranulation probabilistic models are explored in detail.

Proposition 1. Suppose $R_{i}$ is an IN relation over two universes over $U \times V$. For any $E, F \in I N(V), x \in U$, $y \in V$, the two IN thresholds are denoted by $\alpha$ and $\beta$ with $\alpha>\beta$, then the lower and upper approximations for optimistic multigranulation probabilistic models own the following properties:

1. $\sum_{i=1}^{m} R_{i}^{\Omega, \alpha}(\varnothing)=\varnothing, \overline{\sum_{i=1}^{m} R_{i}}(V)=U$

2. $\alpha_{1} \leq \alpha_{2} \Rightarrow \sum_{i=1}^{m} R_{i}^{\Omega, \alpha_{2}}(E) \subseteq \sum_{i=1}^{m} R_{i}^{\Omega, \alpha_{1}}(E), \beta_{1} \leq \beta_{2} \Rightarrow \overline{\sum_{i=1}^{m} R_{i}} \quad \Omega, \beta_{2} \quad(E) \subseteq \overline{\sum_{i=1}^{m} R_{i}} \quad(E)$;

3. $E \subseteq F \Rightarrow \sum_{i=1}^{m} R_{i}^{\Omega, \alpha}(E) \subseteq \sum_{i=1}^{m} R_{i}^{\Omega, \alpha}(F), \overline{\sum_{i=1}^{m} R_{i}} \quad(E) \subseteq \overline{\sum_{i=1}^{m} R_{i}} \quad(F)$;

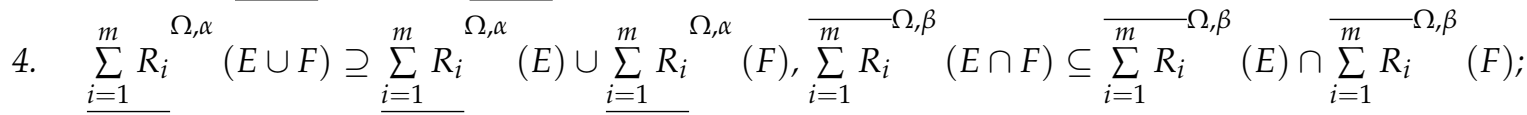

5. ${\overline{\sum_{i=1}^{m} R_{i}}}^{\Omega, \alpha}(E \cap F) \subseteq \overline{\sum_{i=1}^{m} R_{i}}{ }^{\Omega, \alpha}(E) \cap \overline{\sum_{i=1}^{m} R_{i}}{ }^{\Omega, \alpha}(F), \overline{\sum_{i=1}^{m} R_{i}} \quad(E \cup F) \supseteq \overline{\sum_{i=1}^{m} R_{i}} \quad(E) \cup \overline{\sum_{i=1}^{m} R_{i}} \quad(F)$.

Proof.

1. $\sum_{i=1}^{m} R_{i}^{\Omega, \alpha}(\varnothing)=\left\{\Omega_{\varnothing}^{\sum_{i=1}^{m} R_{i}}(x) \geq \alpha \mid x \in U\right\}=\left\{\max _{i=1}^{m} \frac{\sum_{y \in V} R_{i}(x, y) \varnothing}{\sum_{y \in V} R_{i}(x, y)} \geq \alpha \mid x \in U\right\}=\varnothing, \sum_{i=1}^{m} R_{i} \quad(V)=$ $\left\{\Omega_{V}^{i=1} R_{i}^{m}(x)>\beta \mid x \in U\right\}=\left\{\max _{i=1}^{m}\right.$
$\overline{\sum_{i=1}^{m} R_{i}} \Omega, \beta$

2. Since $\alpha_{1} \leq \alpha_{2}$, we have $\sum_{i=1}^{m} R_{i} \Omega^{, \alpha_{2}}(E)=\left\{\Omega_{E}^{\sum_{i=1}^{m} R_{i}}(x) \geq \alpha_{2} \mid x \in U\right\} \subseteq\left\{\Omega_{E}^{\sum_{i=1}^{m} R_{i}}(x) \geq \alpha_{1} \mid x \in U\right\}=$ $\sum_{i=1}^{m} R_{i}{ }^{\Omega, \alpha_{1}}(E)$. Thus, $\alpha_{1} \leq \alpha_{2} \Rightarrow \sum_{i=1}^{m} R_{i}^{\Omega, \alpha_{2}}(E) \subseteq \sum_{i=1}^{m} R_{i}{ }^{\Omega, \alpha_{1}}(E)$ can be concluded. In a similar manner, we can also prove $\beta_{1} \leq \beta_{2} \Rightarrow \overline{\sum_{i=1}^{m} R_{i}}$

$(E) \subseteq \overline{\sum_{i=1}^{m} R_{i}}$ 
3. Since $E \subseteq F$, we have $\sum_{i=1}^{m} R_{i}{ }^{\Omega, \alpha}(E)=\left\{\Omega_{E}^{\sum_{i=1}^{m} R_{i}}(x) \geq \alpha \mid x \in U\right\}=$ $\left\{\max _{i=1} \frac{\sum_{y \in V} R_{i}(x, y) E(y)}{\sum_{y \in V} R_{i}(x, y)} \geq \alpha \mid x \in U\right\} \quad \subseteq$ $\left\{\Omega_{F}^{\sum_{i=1}^{m} R_{i}}(x) \geq \alpha \mid x \in U\right\} \quad=$ $\left\{\max _{i=1}^{m} \frac{\sum_{y \in V} R_{i}(x, y) F(y)}{\sum_{y \in V} R_{i}(x, y)} \geq \alpha \mid x \in U\right\}=\sum_{i=1}^{m} R_{i}^{\Omega, \alpha}(F)$. Thus, $E \subseteq F \Rightarrow \sum_{i=1}^{m} R_{i}^{\Omega, \alpha}(E) \subseteq \sum_{i=1}^{m} R_{i}^{\Omega, \alpha}(F)$

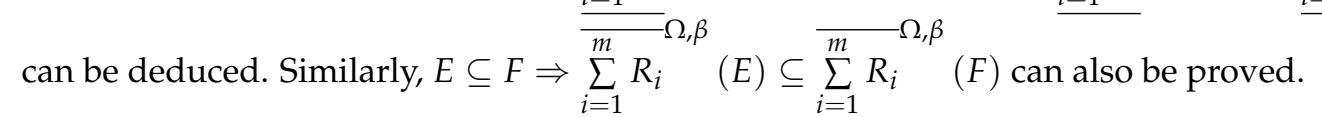

4. $\sum_{i=1}^{m} R_{i}^{\Omega, \alpha}(E \cup F)=\left\{\max _{i=1}^{m} \frac{\sum_{y \in V} R_{i}(x, y)(E \cup F)(y)}{\sum_{y \in V} R_{i}(x, y)} \geq \alpha \mid x \in U\right\} \geq\left\{\max _{i=1}^{m} \frac{\sum_{y \in V} R_{i}(x, y) E(y)}{\sum_{y \in V} R_{i}(x, y)} \geq \alpha \mid x \in U\right\}$, $\overline{i=1}_{i=1}^{m} R_{i}{ }^{2, \alpha}(E \cup F)=\left\{\max _{i=1}^{m} \frac{\sum_{y \in V} R_{i}(x, y)(E \cup F)(y)}{\sum_{y \in V} R_{i}(x, y)} \geq \alpha \mid x \in U\right\} \geq\left\{\max _{i=1}^{m} \frac{\sum_{y \in V} R_{i}(x, y) F(y)}{\sum_{y \in V} R_{i}(x, y)} \geq \alpha \mid x \in U\right\}$. Thus, $\sum_{i=1}^{m} R_{i}^{\Omega, \alpha}(E \cup F) \supseteq \sum^{m} R_{i}^{\Omega, \alpha}(E) \cup \sum_{i=1}^{m} R_{i}{ }^{\Omega, \alpha}(F)$ can be concluded. Similarly, we can also prove $\sum_{i=1}^{\frac{i=1}{m} R_{i}, \beta}(E \cap F) \subseteq \sum_{i=1}^{\overline{\sum_{i=1}^{m} R_{i}}} \Omega, \beta(E) \cap \sum_{i=1}^{\frac{\sum_{i=1}^{m} R_{i}}{L}, \beta}(F)$.

5. According to the above conclusions, $\sum_{i=1}^{m} R_{i}{ }^{\Omega, \alpha}(E \cap F) \subseteq \sum_{i=1}^{m} R_{i}{ }^{\Omega, \alpha}(E) \cap \sum_{i=1}^{m} R_{i}{ }^{\Omega, \alpha}(F)$ and

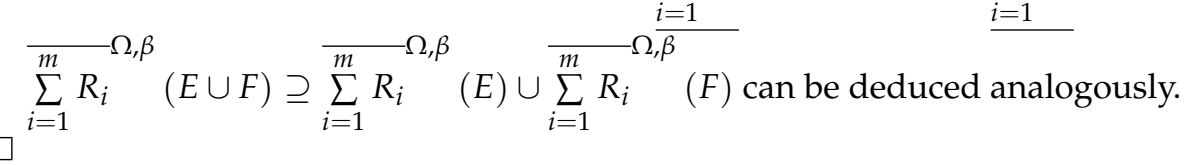

\subsection{Pessimistic IN MG-PRSs over Two Universes}

According to previous definitions, starting from minimal IN membership degrees, pessimistic multigranulation probabilistic models can be established in a similar way.

Definition 9. Suppose $R_{i}$ is an IN relation over two universes over $U \times V$. For any $E \in I N(V), x \in U$, $y \in V$, the two IN thresholds are represented by $\alpha$ and $\beta$ with $\alpha>\beta$, then the lower and upper approximations of $E$ in pessimistic multigranulation probabilistic models are provided as the following mathematical expressions:

$$
\begin{aligned}
& \underline{\sum_{i=1}^{m} R_{i}}{ }^{\Psi, \alpha}(E)=\left\{\Psi_{E}^{\sum_{i=1}^{m} R_{i}}(x) \geq \alpha \mid x \in U\right\} ; \\
& \overline{\sum_{i=1}^{m} R_{i}} \Psi, \beta \quad(E)=\left\{\Psi_{E}^{m} R_{i=1}^{m}(x)>\beta \mid x \in U\right\},
\end{aligned}
$$

the pair $\left(\sum_{i=1}^{m} R_{i}^{\Psi, \alpha}(E),{\overline{\sum_{i=1}^{m} R_{i}}}_{, \mathcal{\beta}}(E)\right)$ is named a pessimistic IN MG-PRS over two universes of $E$.

Next, some key properties of lower and upper approximations for pessimistic multigranulation probabilistic models are presented, and we can prove them according to above-mentioned proofs for Proposition 1.

Proposition 2. Suppose $R_{i}$ is an IN relation over two universes over $U \times V$. For any $E, F \in I N(V), x \in U$, $y \in V$, the two IN thresholds are denoted by $\alpha$ and $\beta$ with $\alpha>\beta$, then the lower and upper approximations for pessimistic multigranulation probabilistic models own the following properties:

1. $\quad{\underline{\sum_{i=1}^{m} R_{i}}}_{, \alpha}(\varnothing)=\varnothing, \sum_{i=1}^{m} R_{i}, \beta(V)=U$ 


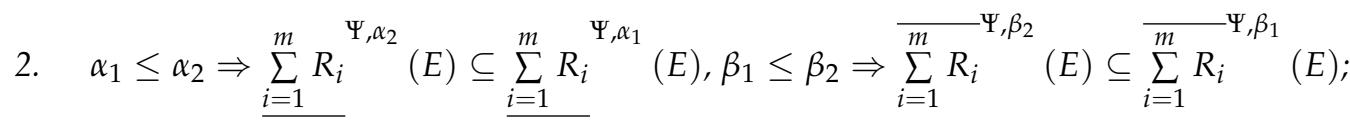

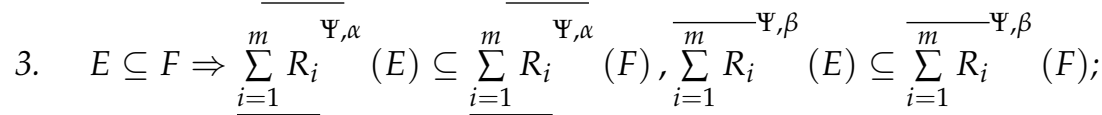

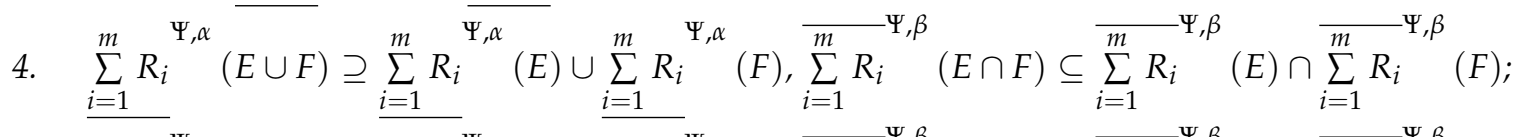

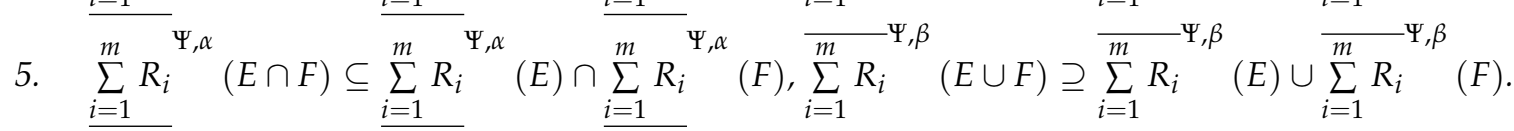

\subsection{Adjustable IN MG-PRSs over Two Universes}

Starting from two mathematical formulations of optimistic and pessimistic multigranulation probabilistic models, the former one utilizes maximal IN membership degrees $\Omega_{E}^{\sum_{i=1}^{m} R_{i}}(x)$ to establish related IN approximations, which indicates a risk-seeking information fusion tactic in MAGDM issues. On the contrary, the latter one utilizes minimal IN membership degrees $\Psi_{E}^{\sum_{i=1}^{m}} R_{i}(x)$ to establish related IN approximations, which indicates a risk-averse information fusion tactic in MAGDM issues. However, it is noted that both optimistic and pessimistic information fusion tactics are qualitative and static, they lack the ability of expressing risks of information fusion tactics from quantitative and dynamic standpoints. In order to quantitatively and dynamically describe the risk preference of information fusion tactics, adjustable IN membership degrees should be put forward by introducing the notion of risk coefficients, then adjustable IN MG-PRSs over two universes can be developed conveniently.

In what follows, adjustable IN membership degrees are defined by means of $\Omega_{E}^{\sum_{i=1}^{m} R_{i}}(x)$ and $\Psi_{E}^{\sum_{i=1}^{m} R_{i}}(x)$

Definition 10. Suppose $R_{i}$ is an IN relation over two universes over $U \times V, \lambda(\lambda \in[0,1])$ is a risk coefficient. For any $E \in I N(V), x \in U, y \in V$, the adjustable IN membership degrees of $x$ in $E$ with regard to $R_{i}$ are provided as the following mathematical expressions:

$$
\Xi_{E}^{\sum_{i=1}^{m} R_{i}}(x)=\lambda \Omega_{E}^{\sum_{i=1}^{m} R_{i}}(x) \boxplus(1-\lambda) \Psi_{E}^{\sum_{i=1}^{m} R_{i}}(x) .
$$

Next, adjustable multigranulation probabilistic models can be designed similarly.

Definition 11. Suppose $R_{i}$ is an $I N$ relation over two universes over $U \times V$. For any $E \in I N(V), x \in U$, $y \in V$, the two IN thresholds are represented by $\alpha$ and $\beta$ with $\alpha>\beta$, then the lower and upper approximations of $E$ in adjustable multigranulation probabilistic models are provided as the following mathematical expressions:

$$
\begin{aligned}
& \sum_{i=1}^{m} R_{i}^{\Xi, \alpha}(E)=\left\{\Xi_{E}^{\sum_{i=1}^{m} R_{i}}(x) \geq \alpha \mid x \in U\right\} ; \\
& \overline{\sum_{i=1}^{m} R_{i}}(E)=\left\{\Xi_{E}^{\sum_{i=1}^{m} R_{i}}(x)>\beta \mid x \in U\right\},
\end{aligned}
$$

the pair $\left(\sum_{i=1}^{m} R_{i}^{\Xi, \alpha}(E), \sum_{i=1}^{m} R_{i}^{\Xi, \beta}(E)\right)$ is named an adjustable IN MG-PRS over two universes of $E$. 
In what follows, some key properties of lower and upper approximations for adjustable multigranulation probabilistic models are presented, and we can also prove them according to above-mentioned proofs for Proposition 1.

Proposition 3. Suppose $R_{i}$ is an IN relation over two universes over $U \times V$. For any $E, F \in I N(V), x \in U$, $y \in V$, the two IN thresholds are denoted by $\alpha$ and $\beta$ with $\alpha>\beta$, then the lower and upper approximations for adjustable multigranulation probabilistic models own the following properties:

1. $\sum_{i=1}^{m} R_{i}^{\Xi, \alpha}(\varnothing)=\varnothing, \sum_{i=1}^{m} R_{i} \Xi, \beta(V)=U ;$

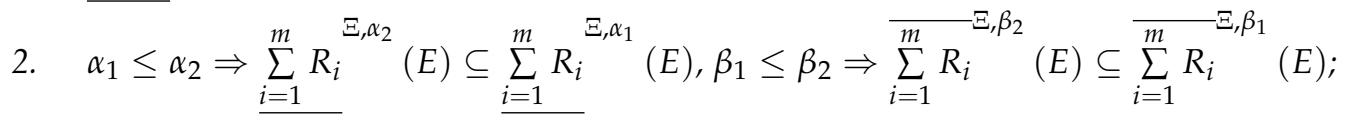

3. $E \subseteq F \Rightarrow \sum_{i=1}^{m} R_{i}^{\Xi, \alpha}(E) \subseteq \sum_{i=1}^{m} R_{i}^{\Xi, \alpha}(F), \overline{\sum_{i=1}^{m} R_{i}} \Xi, \beta(E) \subseteq \overline{\sum_{i=1}^{m} R_{i}}{ }^{\Xi, \beta}(F)$;

4. $\quad \sum_{i=1}^{m} R_{i}^{\Xi, \alpha}(E \cup F) \supseteq \sum_{i=1}^{m} R_{i}^{\overline{\Xi, \alpha}}(E) \cup \sum_{i=1}^{m} R_{i}^{\Xi, \alpha}(F), \overline{\sum_{i=1}^{m} R_{i}} \Xi, \beta \quad(E \cap F) \subseteq \overline{\sum_{i=1}^{m} R_{i}} \Xi, \beta \quad(E) \cap \overline{\sum_{i=1}^{m} R_{i}} \Xi, \beta \quad(F)$;

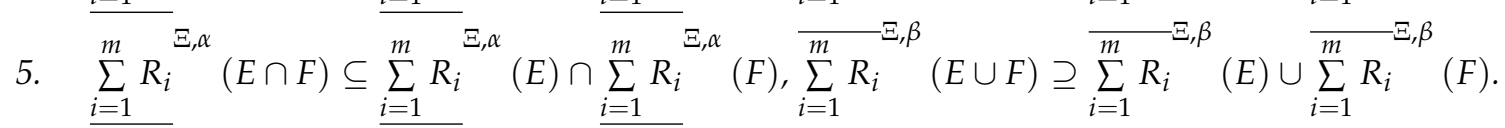

\subsection{Relationships between Optimistic, Pessimistic, and Adjustable IN MG-PRSs over Two Universes}

In previous sections, three types of multigranulation probabilistic models with IN Information are investigated in detail. The following section aims to discuss relationships between optimistic, pessimistic, and adjustable multigranulation probabilistic models.

Proposition 4. Suppose $R_{i}$ is an IN relation over two universes over $U \times V$. For any $E, F \in I N(V), x \in U$, $y \in V$, the two IN thresholds are denoted by $\alpha$ and $\beta$ with $\alpha>\beta$; then, we have:
1. $\sum_{i=1}^{m} R_{i}^{\Psi, \alpha}(E) \subseteq \sum_{i=1}^{m} R_{i}^{\Xi, \alpha}(E) \subseteq \sum_{i=1}^{m} R_{i}^{\Omega, \alpha}(E)$;

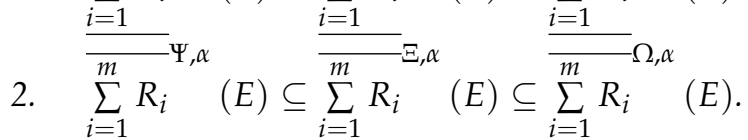

Proof.

1. $\sum_{i=1}^{m} R_{i}^{\Omega, \alpha}(E)=\left\{\Omega_{E}^{\sum_{i=1}^{m} R_{i}}(x) \geq \alpha \mid x \in U\right\}=\left\{\max _{i=1}^{m} \eta_{E}^{R_{i}}(x) \geq \alpha \mid x \in U\right\} \geq$

$\left\{\left(\lambda \max _{i=1}^{m} \eta_{E}^{R_{i}}(x) \boxplus(1-\lambda) \min _{i=1}^{m} \eta_{E}^{R_{i}}(x)\right) \geq \alpha \mid x \in U\right\}=\frac{\sum_{i=1}^{m} R_{i}^{\Xi, \alpha}}{\Psi_{\Psi, \alpha}}(E) \geq$

$\left\{\min _{i=1}^{m} \eta_{E}^{R_{i}}(x) \geq \alpha \mid x \in U\right\}=\sum_{i=1}^{m} R_{i}^{\Psi, \alpha}(E)$. Thus, $\sum_{i=1}^{m} R_{i}^{\overline{\Psi, \alpha}}(E) \subseteq \sum_{i=1}^{m} R_{i}^{\Xi, \alpha}(E) \subseteq \sum_{i=1}^{m} R_{i}^{\Omega, \alpha}(E)$ can

be obtained.

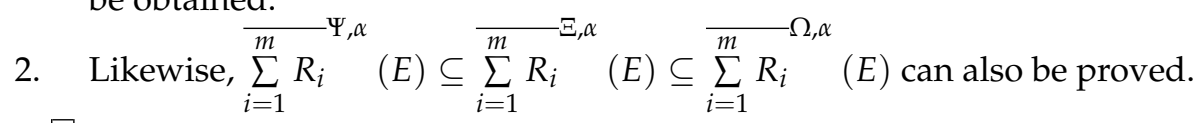

\section{IN MAGDM Based on Multigranulation Probabilistic Models}

In the following section, we aim to sum up a viable and effective MAGDM approach by using the newly developed theoretical models. As pointed out in Section 1, multigranulation probabilistic models can manage the three challenges of typical MAGDM situations well-to be specific, the overall study context of IN MG-PRSs over two universes is IN information, which excels in depicting indeterminate 
and incomplete information at the same time. In addition, the development of multigranulation probabilistic models provide decision makers with an effective strategy in MAGDM information fusion and analysis by taking superiorities of MGRSs and PRSs, and the proposed IN MG-PRSs over two universes are also equipped with the ability of describing risk preferences of information fusion quantitatively and dynamically. Hence, IN MG-PRSs over two universes play a significant role in solving MAGDM problems, and it is necessary to put forward corresponding MAGDM methods.

Next, for the sake of exploring MAGDM methods in a real-world scenario, we put the following discussions in the background of M\&A TSs. We first let the universe $U=\left\{x_{1}, x_{2}, \ldots, x_{j}\right\}$ be a set containing selectable M\&A targets, whereas universe $V=\left\{y_{1}, y_{2}, \ldots, y_{k}\right\}$ is a set containing assessment criteria. Then, we also let $E \in I N(V)$ be a standard set containing several needs of corporate acquirers from the aspect of assessment criteria. Afterwards, $m$ decision makers in a group provide several relations $R_{i} \in I N R(U \times V)(i=1,2, \ldots, m)$ between the above-mentioned universes. Finally, an information system $\left(U, V, R_{i}, E\right)$ for M\&A TSs can be established as the input for the following MAGDM algorithm based on multigranulation probabilistic models.

Remark 1. In what follows, we intend to interpret the scheme of selecting the parametric value $\lambda$. According to Definition 10, the adjustable IN membership degrees of $x$ in E with regard to $R_{i}$ are provided as $\Xi_{E}^{\sum_{i=1}^{m} R_{i}}(x)=$ $\lambda \Omega_{E}^{\sum_{i=1}^{m} R_{i}}(x) \boxplus(1-\lambda) \Psi_{E}^{i=1} R_{i}^{m}(x)$. It is not difficult to see $\Xi_{E}^{\sum_{i=1}^{m} R_{i}}(x)=\Omega_{E}^{\sum_{i=1}^{m} R_{i}}(x)$ when $\lambda=1$, whereas $\sum_{E^{i=1}}^{m} R_{i}(x)=\Psi^{\sum_{i=1}^{m} R_{i}}(x)$ when $\lambda=0$. Hence, maximal IN membership degrees $\Omega_{E}^{\sum_{i=1}^{m} R_{i}}$

$\Xi_{E}^{i=1}(x)=\Psi_{E}^{i=1}(x)$ when $\lambda=0$. Hence, maximal IN membership degrees $\Omega_{E}^{i=1}(x)$ and minimal IN membership degrees $\Psi_{E}^{\sum_{i=1}^{m} R_{i}}(x)$ are two extreme cases of adjustable IN membership degrees $\Xi_{E}^{\sum_{i=1}^{m} R_{i}}(x)$. In light of the standpoint of risk decision-making with uncertainty from classical operational research [1], $\Xi_{E}^{\sum_{i=1}^{m} R_{i}}(x)=\Omega_{E}^{\sum_{i=1}^{m} R_{i}}(x)$ can be seen as the "completely risk-seeking" strategy, $\Xi_{E}^{\sum_{i=1}^{m} R_{i}}(x)=\Psi_{E}^{\sum_{i=1}^{m} R_{i}}(x)$ can be seen as the "completely risk-averse" strategy, and $\Xi_{E}^{i=1} R_{i}^{m}(x)=0.5 \Omega_{E}^{i=1} R_{i}^{m}(x) \boxplus(1-0.5) \Psi_{E}^{i=1} R_{i}^{m}(x)$ can be seen as the "risk-neutral" strategy. Moreover, $\Xi_{E}^{\sum_{i=1}^{m} R_{i}}(x)$ can be seen as the "somewhat risk-seeking" strategy when $\lambda \in(0.5,1)$, and $\Xi_{E}^{\sum_{i=1}^{m} R_{i}}(x)$ can be seen as the "somewhat risk-averse" strategy when $\lambda \in(0,0.5)$. According to the above-stated theoretical explanations, the parametric value $\lambda$ represents the risk preference of different decision makers in MEA TSs. In specific, the larger of the parametric value $\lambda$ when all decision makers are more risk-seeking, whereas the smaller of the parametric value $\lambda$ when all decision makers are more risk-averse. In general, the parametric value $\lambda$ is determined by all decision-makers' risk preference or the empirical studies and inherent knowledge in advance. In practical MAGDM situations, suppose there are $m$ decision makers in a group, each decision maker provides his or her risk preference $\lambda_{i}\left(\lambda_{i} \in[0,1], i=1,2, \ldots, m\right)$, and then the final risk preference when computing $\Xi_{E}^{\sum_{i=1}^{m} R_{i}}(x)$ can be determined as $\lambda=\frac{\sum_{i=1}^{m} \lambda_{i}}{m}$.

In what follows, an algorithm for M\&A TSs by virtue of adjustable IN MG-PRSs over two universes is established.

\section{An Illustrative Example}

For the sake of making an efficient comparative analysis with existing similar IN MAGDM approaches, we plan to utilize the case study that was previously investigated in [28]. In what follows, we first present the general context of M\&A TSs and show basic steps of obtaining the optimal M\&A target by means of the newly proposed algorithm developed in Section 4 . 
Remark 2. Section 4 acts as a transition part which links between the theoretical models proposed in Section 3 and the application case presented in Section 5. To be specific, we first put forward two special theoretical models named optimistic and pessimistic IN MG-PRSs over two universes. Then, we further generalize these two special theoretical models to adjustable IN MG-PRSs over two universes. All the proposed three theoretical models are foundations for addressing MAGDM problems. Next, we propose a novel algorithm for MEA TSs in light of adjustable IN MG-PRSs over two universes in Section 4. Finally, in order to show the reasonability and effectiveness of the proposed algorithm, the following section plans to conduct several quantitative and qualitative analysis via an illustrative example.

\subsection{MAGDM Procedures}

In this illustrative example, we use the case study that was previously investigated in [28]. The detailed case descriptions and datasets can be found at the webpage (https:/ / www.mdpi.com/2073-8994/9/7/126/htm).

According to Algorithm 1, we aim to obtain the optimal M\&A target by means of IN MG-PRSs over two universes. First, we calculate single IN membership degrees as follows.

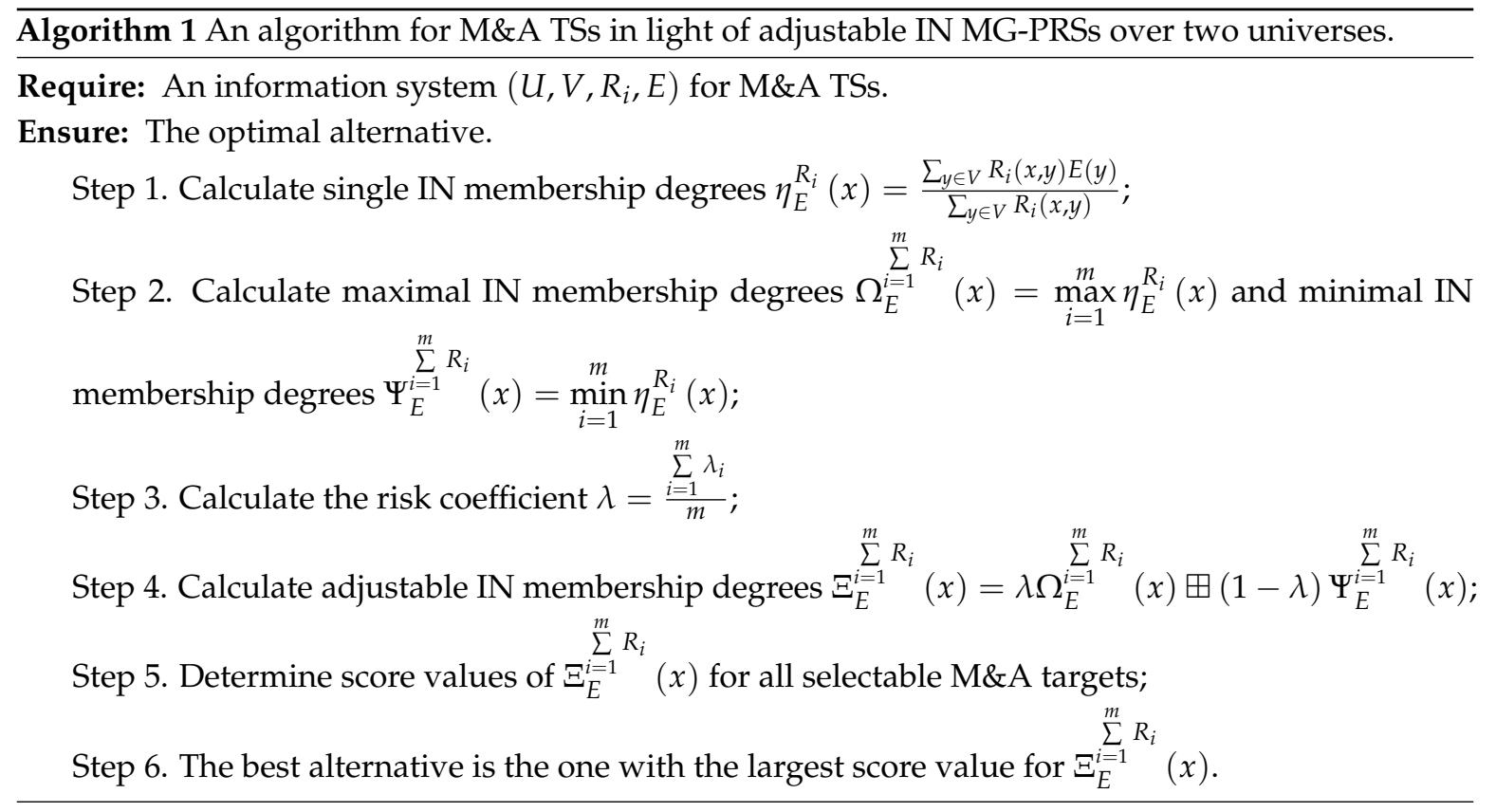

With regard to the relation presented in $R_{1}$, we obtain

$\eta_{E}^{R_{1}}\left(x_{1}\right)=\frac{\sum_{y \in V} R_{1}\left(x_{1}, y\right) E(y)}{\sum_{y \in V} R_{1}\left(x_{1}, y\right)}=\langle[0.8477,0.9207],[0.0378,0.1345],[0.1771,0.316]\rangle$.

In a similar manner, we also obtain

$$
\begin{aligned}
& \eta_{E}^{R_{1}}\left(x_{2}\right)=\langle[0.7672,0.894],[0.0459,0.1677],[0.3197,0.5164]\rangle ; \\
& \eta_{E}^{R_{1}}\left(x_{3}\right)=\langle[0.8212,0.9314],[0.05,0.1497],[0.2351,0.3925]\rangle ; \\
& \eta_{E}^{R_{1}}\left(x_{4}\right)=\langle[0.8493,0.9465],[0.0232,0.101],[0.2147,0.4122]\rangle ; \\
& \eta_{E}^{R_{1}}\left(x_{5}\right)=\langle[0.8774,0.9643],[0.0466,0.1559],[0.07,0.1923]\rangle
\end{aligned}
$$


With regard to the relation presented in $R_{2}$, we also obtain

$\eta_{E}^{R_{2}}\left(x_{1}\right)=\langle[0.8185,0.9419],[0.033,0.1355],[0.17,0.3418]\rangle ;$

$\eta_{E}^{R_{2}}\left(x_{2}\right)=\langle[0.7728,0.9177],[0.04,0.1423],[0.285,0.4756]\rangle$;

$\eta_{E}^{R_{2}}\left(x_{3}\right)=\langle[0.8217,0.9407],[0.038,0.1294],[0.1799,0.3357]\rangle ;$

$\eta_{E}^{R_{2}}\left(x_{4}\right)=\langle[0.8327,0.9548],[0.022,0.097],[0.2434,0.4106]\rangle ;$

$\eta_{E}^{R_{2}}\left(x_{5}\right)=\langle[0.8591,0.9645],[0.031,0.1583],[0.096,0.2316]\rangle$.

With regard to the relation presented in $R_{3}$, we also obtain

$\eta_{E}^{R_{3}}\left(x_{1}\right)=\langle[0.844,0.9472],[0.03,0.1422],[0.2104,0.3615]\rangle ;$

$\eta_{E}^{R_{3}}\left(x_{2}\right)=\langle[0.799,0.91],[0.0336,0.1571],[0.3151,0.5472]\rangle ;$

$\eta_{E}^{R_{3}}\left(x_{3}\right)=\langle[0.8388,0.9368],[0.0424,0.1466],[0.1803,0.3484]\rangle ;$

$\eta_{E}^{R_{3}}\left(x_{4}\right)=\langle[0.8362,0.9366],[0.0188,0.1069],[0.206,0.3834]\rangle$;

$\eta_{E}^{R_{3}}\left(x_{5}\right)=\langle[0.8739,0.9581],[0.029,0.1351],[0.075,0.2316]\rangle$.

Next, maximal and minimal IN membership degrees can be calculated in light of Definition 7. For maximal IN membership degrees, we have

$\Omega_{E}^{\sum_{i=1}^{3} R_{i}}\left(x_{1}\right)=\langle[0.8477,0.9472],[0.0378,0.1422],[0.2104,0.3615]\rangle ;$

$\Omega_{E}^{\sum_{i=1}^{3} R_{i}}\left(x_{2}\right)=\langle[0.799,0.9177],[0.0459,0.1677],[0.3197,0.5472]\rangle$;

$\Omega_{E}^{\sum_{i=1}^{3} R_{i}}\left(x_{3}\right)=\langle[0.8388,0.9407],[0.05,0.1497],[0.2351,0.3925]\rangle$;

$\Omega_{E}^{\sum_{i=1}^{3} R_{i}}\left(x_{4}\right)=\langle[0.8493,0.9548],[0.0232,0.1069],[0.2434,0.4122]\rangle$;

$\Omega_{E}^{\sum_{i=1}^{3} R_{i}}\left(x_{5}\right)=\langle[0.8774,0.9645],[0.0466,0.1583],[0.096,0.2316]\rangle$.

Similarly, for minimal IN membership degrees, we also have

$\Psi_{E}^{\sum_{i=1}^{3} R_{i}}\left(x_{1}\right)=\langle[0.8185,0.9207],[0.03,0.1345],[0.17,0.316]\rangle ;$

$\Psi_{E}^{\sum_{i=1}^{3} R_{i}}\left(x_{2}\right)=\langle[0.7672,0.894],[0.0336,0.1423],[0.285,0.4756]\rangle ;$

$\Psi_{E}^{\sum_{i=1}^{3} R_{i}}\left(x_{3}\right)=\langle[0.8212,0.9314],[0.038,0.1294],[0.1799,0.3357]\rangle ;$

$\Psi_{E}^{\sum_{i=1}^{3} R_{i}}\left(x_{4}\right)=\langle[0.8327,0.9366],[0.0188,0.097],[0.206,0.3834]\rangle$;

$\Psi_{E}^{\sum_{i=1}^{3} R_{i}}\left(x_{5}\right)=\langle[0.8591,0.9581],[0.029,0.1351],[0.07,0.1923]\rangle$.

In order to make an efficient comparison with the MAGDM method proposed in [28], the risk coefficient $\lambda=0.6$ is noted in [28]; then, we also take the risk coefficient $\lambda=0.6$ in this case study. In 
what follows, adjustable IN membership degrees can be calculated. To be specific, for M\&A target $x_{1}$, we have

$$
\Xi_{E}^{\sum_{i=1}^{3} R_{i}}\left(x_{1}\right)=0.6 \Omega_{E}^{\sum_{i=1}^{3} R_{i}}\left(x_{1}\right) \boxplus(1-0.6) \Psi_{E}^{\sum_{i=1}^{3} R_{i}}\left(x_{1}\right)=\langle[0.8366,0.9379],[0.0345,0.1391],[0.1932,0.3426]\rangle .
$$

In what follows, we also have

$$
\begin{aligned}
& \sum_{E}^{\sum_{i=1}^{3} R_{i}}\left(x_{2}\right)=\langle[0.7868,0.9089],[0.0405,0.157],[0.3054,0.5173]\rangle ; \\
& \Xi_{E}^{\sum_{i=1}^{3} R_{i}}\left(x_{3}\right)=\langle[0.832,0.9371],[0.0448,0.1412],[0.2112,0.3687]\rangle ; \\
& \Xi_{E}^{\sum_{i=1}^{3} R_{i}}\left(x_{4}\right)=\langle[0.8429,0.9482],[0.0213,0.1028],[0.2277,0.4004]\rangle ; \\
& \Xi_{E}^{\sum_{i=1}^{3} R_{i}}\left(x_{5}\right)=\langle[0.8705,0.9621],[0.0385,0.1486],[0.0846,0.215]\rangle .
\end{aligned}
$$

Finally, we calculate score values of $\Xi_{E}^{3} \sum_{i=1}^{3} R_{i}(x)$ for each $x_{i}$, and the best $x_{i}$ is the one with the largest score value for $\Xi^{\sum_{i=1}^{3}} R_{i}$ alternative is $x_{5}$.

\subsection{Sensitivity Analysis}

In the previous section, we obtain the optimal M\&A target by using adjustable multigranulation probabilistic models with the risk coefficient $\lambda=0.6$. The following sensitivity analysis aims to investigate the influence of the risk coefficient by changing the value of $\lambda$. To be specific, supposing the value of $\lambda$ is taken as $0,0.4,0.5,0.6$, and 1 , respectively, then we can obtain the final ranking orders in Table 1 below.

Table 1. Ranking orders of M\&A targets with changing values of $\lambda$.

\begin{tabular}{ccc}
\hline Different $\lambda$ & Information Fusion Risks & Ranking Results of M\&A Targets \\
\hline$\lambda=0$ & completely pessimistic (completely risk-averse) & $x_{5} \succ x_{1} \succ x_{3} \succ x_{4} \succ x_{2}$ \\
$\lambda=0.4$ & somewhat pessimistic (somewhat risk-averse) & $x_{5} \succ x_{1} \succ x_{4} \succ x_{3} \succ x_{2}$ \\
$\lambda=0.5$ & neutral (risk-neutral) & $x_{5} \succ x_{1} \succ x_{4} \succ x_{3} \succ x_{2}$ \\
$\lambda=0.6$ & somewhat optimistic (somewhat risk-seeking) & $x_{5} \succ x_{1} \succ x_{4} \succ x_{3} \succ x_{2}$ \\
$\lambda=1$ & completely optimistic (completely risk-seeking) & $x_{5} \succ x_{1} \succ x_{4} \succ x_{3} \succ x_{2}$ \\
\hline
\end{tabular}

According to the final ranking orders in Table 1 , it is easy to see that the best $x_{i}$ is insensitive to changing values of $\lambda$, that is, all results show the best $x_{i}$ is $x_{5}$. Thus, the best alternative is reliable and stable. The only difference lies in the ranking order of $x_{3}$ and $x_{4}$ when $\lambda=0$, i.e., $x_{3}$ is superior to $x_{4}$ when $\lambda=0$, whereas $x_{3}$ is inferior to $x_{4}$ in other situations. The cause of this phenomenon is that the changing values of $\lambda$ may affect the ranking order of $x_{3}$ and $x_{4}$ when the risk preferences is completely risk-averse.

\subsection{Comparative Analysis}

In what follows, we aim to compare with the MAGDM method proposed in [28] to present the merits of the proposed MAGDM algorithm. In [28], the authors put forward an algorithm for M\&A 
TSs via IN MGRSs over two universes without the support of PRSs. The mathematical structures of optimistic and pessimistic IN MGRSs over two universes are presented below:

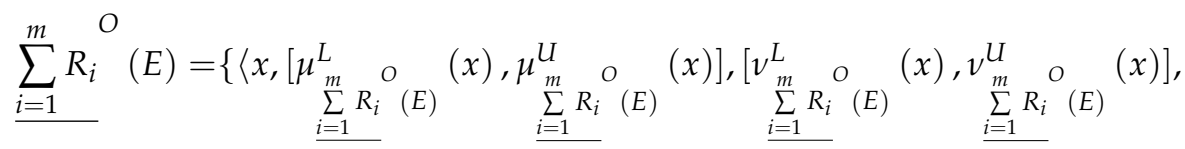

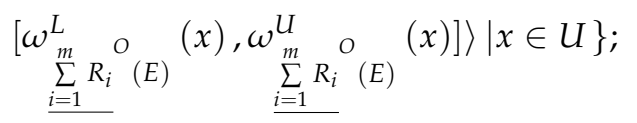

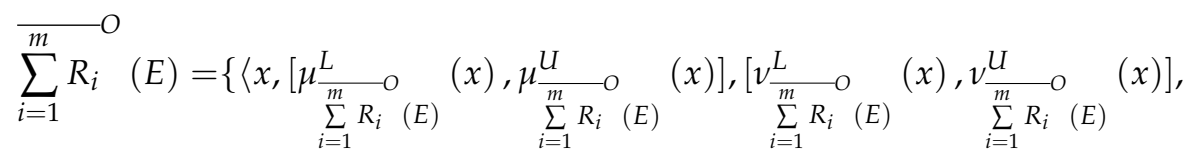

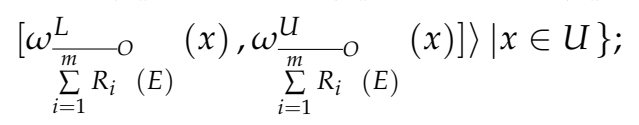

$$
\begin{aligned}
& \underline{\sum_{i=1}^{m} R_{i}^{P}}(E)=\left\{\left\langlex,\left[\mu_{\sum_{i=1}^{L} R_{i}^{P}(E)}^{P}(x), \mu_{\sum_{i=1}^{U} R_{i}^{P}(E)}^{P}(x)\right],\left[v_{\sum_{i=1}^{L} R_{i}^{P}(E)}^{P}(x), v_{\sum_{i=1}^{U} R_{i}^{P}(E)}^{P}(x)\right],\right.\right. \\
& \left.\left[\omega^{\sum_{i=1}^{L} R_{i}^{P}(E)}(x), \omega_{\sum_{i=1}^{U} R_{i}^{P}(E)}^{P}(x)\right]\right\rangle \mid x \in \overline{U\}} ;
\end{aligned}
$$

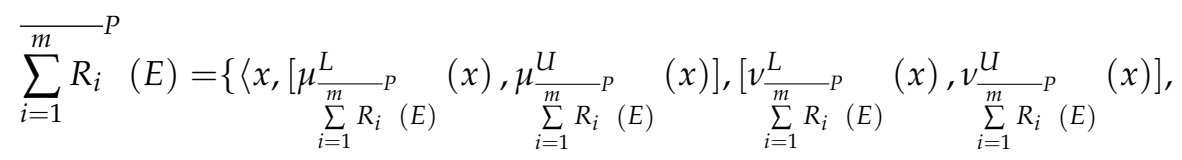

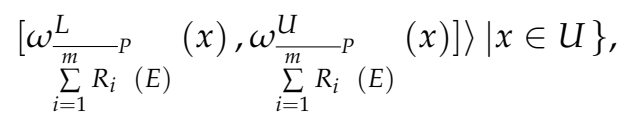

where

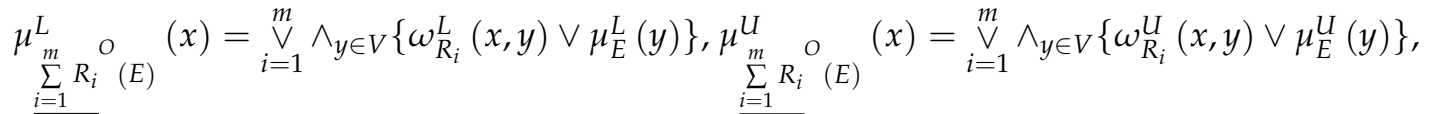

$$
\begin{aligned}
& v_{\sum_{i=1}^{m} R_{i}}^{L_{i}(E)}(x)=\wedge_{i=1}^{m} \vee_{y \in V}\left\{\left(1-v_{R_{i}}^{U}(x, y)\right) \wedge v_{E}^{L}(y)\right\}
\end{aligned}
$$

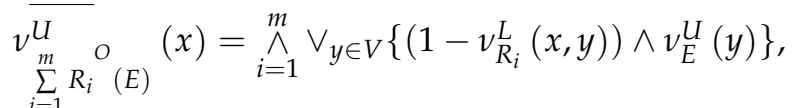

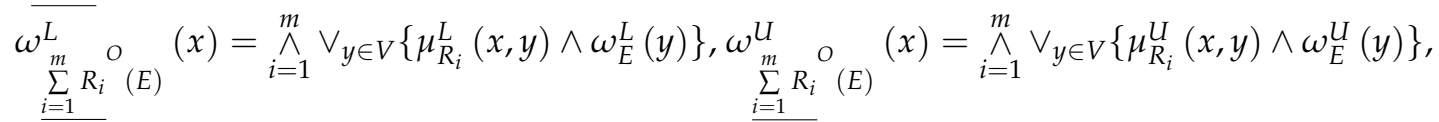

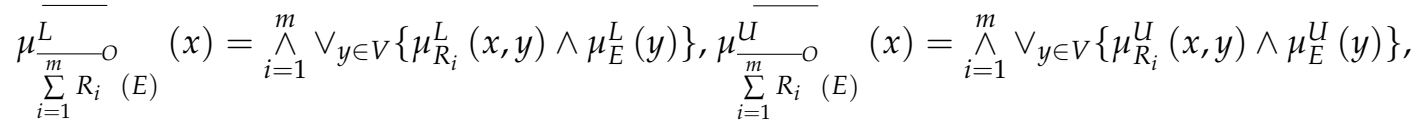

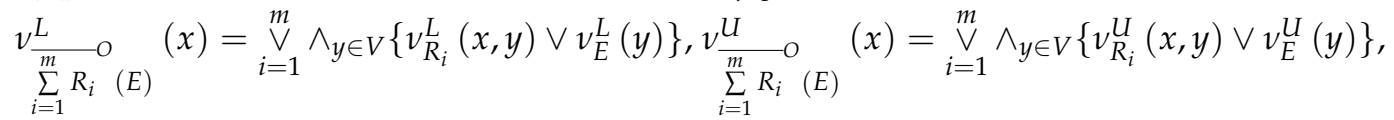

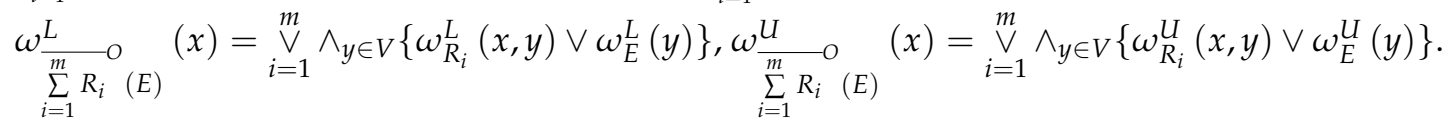

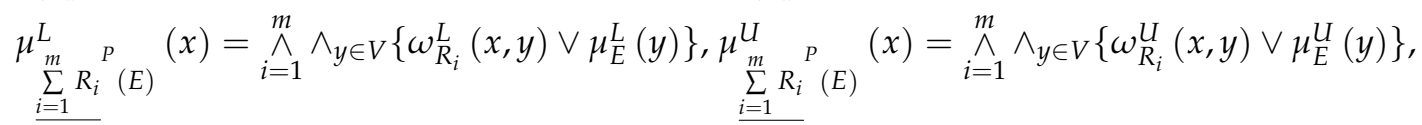




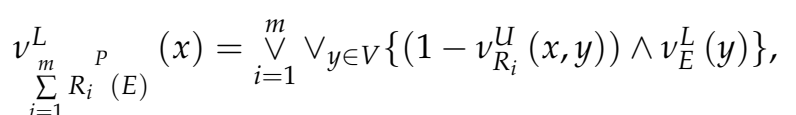

$v_{\sum_{i=1}^{m} R_{i}^{P}(E)}^{\bar{U}}(x)=\bigvee_{i=1}^{m} \vee_{y \in V}\left\{\left(1-v_{R_{i}}^{L}(x, y)\right) \wedge v_{E}^{U}(y)\right\}$

${\overline{\sum_{i=1}^{m} R_{i}{ }^{P}(E)}}^{L}(x)=\bigvee_{i=1}^{m} \vee_{y \in V}\left\{\mu_{R_{i}}^{L}(x, y) \wedge \omega_{E}^{L}(y)\right\}, \omega_{\sum_{i=1}^{m} R_{i}{ }^{P}(E)}(x)=\bigvee_{i=1}^{m} \vee_{y \in V}\left\{\mu_{R_{i}}^{U}(x, y) \wedge \omega_{E}^{U}(y)\right\}$,

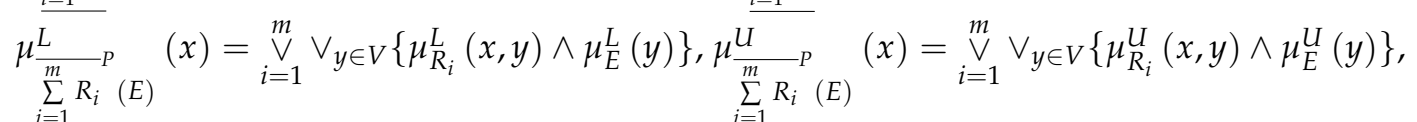

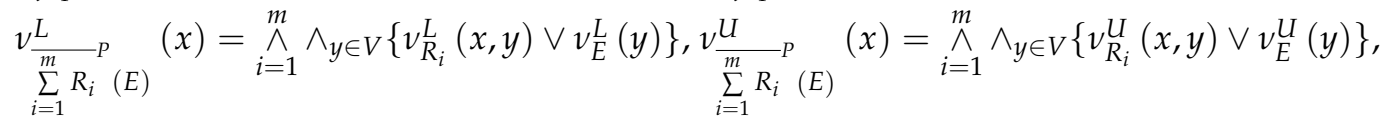

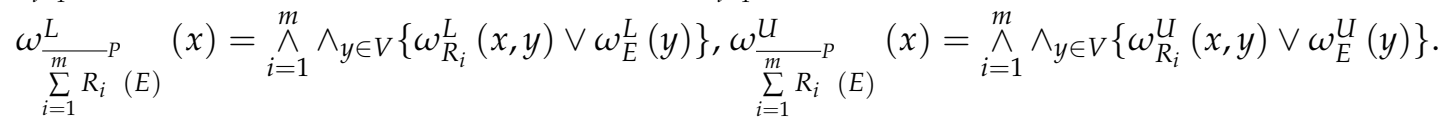

The pair $\left(\sum_{i=1}^{m} R_{i}(E), \overline{\sum_{i=1}^{m} R_{i}}(E)\right)$ is named an optimistic IN MGRS over two universes of $E$, whereas the pair $\left(\sum_{i=1}^{m} R_{i} P(E), \overline{\sum_{i=1}^{m} R_{i}} P(E)\right)$ is named a pessimistic IN MGRS over two universes of $E$.

More concretely, the optimistic and pessimistic IN multigranulation rough approximations in terms of an information system $\left(U, V, R_{i}, E\right)(i=1,2,3)$ for M\&A TSs are calculated at first, which are denoted by $\sum_{i=1}^{3} R_{i}(E), \overline{\sum_{i=1}^{3} R_{i}}(E), \sum_{i=1}^{3} R_{i}(E)$ and $\overline{\sum_{i=1}^{3} R_{i}}(E)$. Then, we further synthesize lower and upper versions of optimistic IN multigranulation rough approximations, which is denoted by $\sum_{i=1}^{3} R_{i} \quad(E) \boxplus \overline{\sum_{i=1}^{3} R_{i}}(E)$, whereas lower and upper versions of pessimistic counterparts can also be synthesized, which is denoted by $\sum_{i=1}^{3} R_{i}(E) \boxplus \overline{\sum_{i=1}^{3} R_{i}} P(E)$. Let the risk coefficient $\lambda=0.6$ when integrating $\sum_{i=1}^{3} R_{i}^{O}(E) \boxplus \overline{\sum_{i=1}^{3} R_{i}} \quad(E)$ with $\sum_{i=1}^{3} R_{i} \quad(E) \boxplus \overline{\sum_{i=1}^{3} R_{i}} P(E)$, i.e., the synthesized set $0.6\left(\sum_{i=1}^{3} R_{i}^{O}(E) \boxplus \overline{\sum_{i=1}^{3} R_{i}}(E)\right) \boxplus(1-0.6)\left(\sum_{i=1}^{3} R_{i}{ }^{P}(E) \boxplus \overline{\sum_{i=1}^{3} R_{i}} P(E)\right)$ should be further obtained. The above-stated sets are obtained as follows:

$\sum_{i=1}^{3} R_{i} \quad(E) \boxplus{\overline{\sum_{i=1}^{3} R_{i}}}^{O}(E)=\left\{\left\langle x_{1},\langle[0.82,0.90],[0.15,0.24],[0.24,0.35]\rangle\right\rangle\right.$

$\left\langle x_{2},\langle[0.70,0.80],[0.15,0.35],[0.42,0.56]\rangle\right\rangle,\left\langle x_{3},\langle[0.85,0.92],[0.08,0.18],[0.30,0.42]\rangle\right\rangle$,

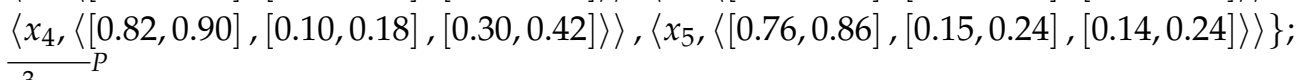

$\sum_{i=1}^{3} R_{i}^{P}(E) \boxplus{\overline{\sum_{i=1}^{3} R_{i}}}^{P}(E)=\left\{\left\langle x_{1},\langle[0.82,0.90],[0.10,0.21],[0.24,0.35]\rangle\right\rangle\right.$,

$\left\langle x_{2},\langle[0.70,0.85],[0.15,0.28],[0.40,0.54]\rangle\right\rangle,\left\langle x_{3},\langle[0.85,0.92],[0.08,0.18],[0.24,0.35]\rangle\right\rangle$,

$\left.\left\langle x_{4},\langle[0.79,0.88],[0.10,0.21],[0.28,0.40]\rangle\right\rangle,\left\langle x_{5},\langle[0.76,0.86],[0.15,0.28],[0.16,0.27]\rangle\right\rangle\right\} ;$

$0.6\left(\sum_{i=1}^{3} R_{i}^{O}(E) \boxplus{\overline{\sum_{i=1}^{3} R_{i}}}^{O}(E)\right) \boxplus(1-0.6)\left(\sum_{i=1}^{3} R_{i}^{P}(E) \boxplus{\overline{\sum_{i=1}^{3} R_{i}}}^{P}(E)\right)=$ $\left\{\left\langle x_{1},\langle[0.82,0.90],[0.13,0.23],[0.24,0.35]\rangle\right\rangle,\left\langle x_{2},\langle[0.70,0.82],[0.15,0.32],[0.41,0.55]\rangle\right\rangle\right.$, $\left\langle x_{3},\langle[0.85,0.92],[0.08,0.18],[0.28,0.39]\rangle\right\rangle,\left\langle x_{4},\langle[0.81,0.89],[0.10,0.19],[0.29,0.41]\rangle\right\rangle$, $\left.\left\langle x_{5},\langle[0.76,0.86],[0.15,0.25],[0.15,0.25]\rangle\right\rangle\right\}$. 
Finally, score values of $0.6\left(\sum_{i=1}^{3} R_{i}^{O} \quad(E) \boxplus \overline{\sum_{i=1}^{3} R_{i}}(E)\right) \boxplus(1-0.6)\left(\sum_{i=1}^{3} R_{i}^{P}(E) \boxplus \overline{\sum_{i=1}^{3} R_{i}}(E)\right)$ are calculated, and it is convenient to determine the ranking orders of five $\overline{M \&} \mathrm{~A}$ targets via the above score values and obtain the optimal M\&A target, which is $x_{3}$, and $x_{5}$ is ranked second. The reason for the difference with the result obtained by using the proposed method lies in IN MGRSs over two universes lacking the ability of error tolerance; the MAGDM result is sensitive to outlier values from original information for M\&A TSs.

\subsection{Discussion}

In order to address complicated MAGDM problems effectively, three key challenges are focused on at first. Then, under the guidance of multigranulation probabilistic models, we utilize the model of INSs, MGRSs and PRSs to handle the above-mentioned challenges. Moreover, compared with existing popular nonlinear modeling approaches, such as formal concept analysis [33,34,58,64-67], control systems [59,60] and sentiment analysis [61-63,68,69], which neither effectively handle indeterminate and incomplete information in complicated MAGDM problems, nor reasonably fuse and analyze multi-source information with incorrect and noisy data, it is necessary to combine INSs, MGRSs with PRSs to develop some meaningful hybrid models along with corresponding MAGDM approaches. In light of MAGDM procedures in the current section, we sum up the merits of the proposed MAGDM algorithm below:

1. INSs act as a viable and effective tool for depicting various uncertainties in typical MAGDM situations. By dividing the notion of membership degrees into three different parts, indeterminate and incomplete MAGDM information can be described precisely.

2. In MAGDM information fusion procedures, with the support of MGRSs, the computational efficiency of information fusion can be enhanced to a large extent. Moreover, decision risks of information fusion strategies can also be modeled well.

3. Compared with [28], the proposed MAGDM algorithm excels in fusing the superiorities of PRSs in the construction of hybrid models. To be specific, IN MG-PRSs over two universes own the fault-tolerance ability when coping with incorrect and noisy data.

Hence, the developed IN MG-PRSs over two universes perform outstandingly in MAGDM information representation, information fusion, and information analysis; they provide a beneficial tool for addressing complicated MAGDM problems.

\section{Conclusions}

This work mainly presents a general framework for dealing with complicated IN MAGDM problems by virtue of multigranulation probabilistic models. At first, three different types of multigranulation probabilistic models are put forward, that is, the optimistic version, the pessimistic version, and the adjustable version, and both definitions and key properties are discussed in detail. Then, relationships between optimistic, pessimistic, and adjustable multigranulation probabilistic models are further explored. Afterwards, corresponding IN MAGDM approaches are proposed in the background of M\&A TSs. Finally, a practical example of M\&A TSs is presented with several quantitative and qualitative analysis.

In the future, it is meaningful to generalize IN MG-PRSs over two universes to more extended neutrosophic contexts such as neutrosophic duplets, triplets and multisets. Furthermore, establishing efficient IN MAGDM approaches for problems with dynamic situations, high-dimensional attributes and large-scale of alternatives are also necessary. Another future interesting study option is to apply the presented theoretical models to other areas such as clustering, feature selections, compressed sensing, image processing, etc. 
Author Contributions: Conceptualization, D.L.; writing-original draft preparation, C.Z.; validation, X.K. and Y.L.; writing-review and editing, S.B. and A.K.S.; supervision, D.L. All authors have read and agreed to the published version of the manuscript.

Funding: This work was partially supported by the Key R\&D program of Shanxi Province (International Cooperation, 201903D421041), National Natural Science Foundation of China (Nos. 61806116, 61672331, 61972238, 61802237, 61603278 and 61906110), Natural Science Foundation of Shanxi (Nos. 201801D221175, 201901D211176, 201901D211414), Training Program for Young Scientific Researchers of Higher Education Institutions in Shanxi, Scientific and Technological Innovation Programs of Higher Education Institutions in Shanxi (STIP) (Nos. 201802014, 2019L0066, 2019L0500), and Cultivate Scientific Research Excellence Programs of Higher Education Institutions in Shanxi (CSREP) (2019SK036).

Acknowledgments: The authors are grateful to the editor and three anonymous reviewers for their helpful and valuable comments which helped to improve the quality of the research.

Conflicts of Interest: The authors declare no conflict of interest.

\section{References}

1. Xu, Z.S. Uncertain Multi-Attribute Decision Making: Methods and Applications; Springer: Berlin, Germany, 2015; pp. 1-373.

2. Sun, B.Z.; Ma, W.M.; Xiao, X. Three-way group decision-making based on multigranulation fuzzy decision-theoretic rough set over two universes. Int. J. Approx. Reason. 2017, 81, 87-102. [CrossRef]

3. Liang, D.C.; Zhang, Y.R.J.; Xu, Z.S.; Darko, A.P. Pythagorean fuzzy Bonferroni mean aggregation operator and its accelerative calculating algorithm with the multithreading. Int. J. Intell. Syst. 2018, 33, $615-633$. [CrossRef]

4. Zhang, C.; Li, D.Y.; Liang, J.Y. Hesitant fuzzy linguistic rough set over two universes model and its applications. Int. J. Mach. Learn. Cyb. 2018, 9, 577-588. [CrossRef]

5. Zhang, K.; Zhan, J.M.; Yao, Y.Y. TOPSIS method based on a fuzzy covering approximation space: An application to biological nano-materials selection. Inform. Sci. 2019, 502, 297-329. [CrossRef]

6. Zhang, C.; Li, D.Y.; Liang, J.Y. Interval-valued hesitant fuzzy multi-granularity three-way decisions in consensus processes with applications to multi-attribute group decision-making. Inform. Sci. 2020, 511, 192-211. [CrossRef]

7. Smarandache, F. Neutrosophy. Neutrosophic Probability, Set, and Logic; American Research Press: Rehoboth, DE, USA, 1998.

8. Smarandache, F. A Unifying Field in Logics. Neutrosophy: Neutrosophic Probability, Set and Logic; American Research Press: Rehoboth, DE, USA, 1999.

9. Peng, X.D.; Dai, J.G. A bibliometric analysis of neutrosophic set: Two decades review from 1998 to 2017. Artif. Intell. Rev. $2018,1-57$. [CrossRef]

10. An, X.G.; Zhang, X.H.; Ma, Y.C. Generalized abel-grassmann's neutrosophic extended triplet loop. Mathematics 2019, 7, 1206. [CrossRef]

11. Guo, Y.H.; Sengur, A. A novel 3D skeleton algorithm based on neutrosophic cost function. Appl. Soft. Comput. 2015, 36, 210-217. [CrossRef]

12. Smarandache, F. Refined neutrosophy and lattices vs. pair structures and yinyang bipolar fuzzy set. Mathematics 2019, 7, 353. [CrossRef]

13. Wang, H.B.; Smarandache, F.; Zhang, Y.Q.; Sunderraman, R. Interval Neutrosophic Sets and Logic: Theory and Applications in Computing; Hexis: Phoenix, AZ, USA, 2005; pp. 1-87.

14. Broumi, S.; Smarandache, F. Correlation coefficient of interval neutrosophic set. Appl. Mech. Mater. 2013, 436, 511-517. [CrossRef]

15. Zhang, H.Y.; Wang, J.Q.; Chen, X.H. Interval neutrosophic sets and their application in multicriteria decision-making problems. Sci. World J. 2014, 2014. [CrossRef] [PubMed]

16. Fan, C.X.; Ye, J.; Feng, S.; Fan, E.; Hu, K.L. Multi-criteria decision-making method using heronian mean operators under a bipolar neutrosophic environment. Mathematics 2019, 7, 97. [CrossRef]

17. Ye, J. Multiple attribute group decision-making based on interval neutrosophic uncertain linguistic variables. Int. J. Mach. Learn. Cyb. 2017, 8, 837-848. [CrossRef] 
18. Deli, I. Interval-valued neutrosophic soft sets and its decision-making. Int. J. Mach. Learn. Cyb. 2017, 8, 665-676. [CrossRef]

19. Li, X.; Zhang, X.H.; Park, C. Generalized interval neutrosophic choquet aggregation operators and their applications. Symmetry 2018, 10, 85. [CrossRef]

20. Nguyen, T.T.; Luu, Q.D.; Le, H.S.; Zhang, X.H.; Nguyen, D.H.; Ali, M.; Smarandache, F. Dynamic interval valued neutrosophic set: Modeling decision-making in dynamic environments. Comput. Ind. 2019, 108, 45-52.

21. Broumi, S.; Talea, M.; Bakali, A.; Smarandache, F.; Gai, S.Q.; Selvachandran, G. Introduction of some new results on interval-valued neutrosophic graphs. J. Inform. Optim. Sci. 2019, 40, 1475-1498. [CrossRef]

22. Rani, D.; Garg, H. Some modified results of the subtraction and division operations on interval neutrosophic sets. J. Exp. Theor. Artif. Intell. 2019, 31, 677-698. [CrossRef]

23. Xu, W.H.; Yu, J.H. A novel approach to information fusion in multi-source datasets: A granular computing viewpoint. Inform. Sci. 2017, 378, 410-423. [CrossRef]

24. Lin, B.Y.; Xu, W.H. Multi-granulation rough set for incomplete interval-valued decision information systems based on multi-threshold tolerance relation. Symmetry 2018, 10, 208. [CrossRef]

25. Broumi, S.; Smarandache, F.; Dhar, M. Rough neutrosophic sets. Ital. J. Pure. Appl. Math. 2014, 32, 493-502.

26. Broumi, S.; Smarandache, F. Soft Interval-valued neutrosophic rough sets. Neutrosophic Sets Syst. 2015, 7, 69-80. [CrossRef]

27. Zhang, C.; Zhai, Y.H.; Li, D.Y.; Mu, Y.M. Steam turbine fault diagnosis based on single-valued neutrosophic multigranulation rough sets over two universes. J. Intell. Fuzzy. Syst. 2016, 31, 2829-2837. [CrossRef]

28. Zhang, C.; Li, D.Y.; Sangaiah, A.; Broumi, S. Merger and acquisition target selection based on interval neutrosophic multigranulation rough sets over two universes. Symmetry 2017, 9, 126. [CrossRef]

29. Liu, Y.L.; Yang, H.L. Multi-granulation neutrosophic rough sets on a single domain and dual domains with applications. Symmetry 2017, 33, 1467-1478.

30. Shao, S.T.; Zhang, X.H. Measures of probabilistic neutrosophic hesitant fuzzy sets and the application in reducing unnecessary evaluation processes. Mathematics 2019, 7, 649. [CrossRef]

31. Yang, H.L.; Bao, Y.L.; Guo, Z.L. Generalized interval neutrosophic rough sets and its application in multi-attribute decision-making. Filomat 2018, 32, 11-33. [CrossRef]

32. Zhang, C.; Li, D.Y.; Kang, X.P.; Song, D.; Sangaiah, A.; Broumi, S. Neutrosophic fusion of rough set theory: An overview. Comput. Ind. 2020, 115, 103117. [CrossRef]

33. Singh, P.K. Three-way fuzzy concept lattice representation using neutrosophic set. Int. J. Mach. Learn. Cyb. 2017, 8, 69-79. [CrossRef]

34. Singh, P.K. Three-way n-valued neutrosophic concept lattice at different granulation. Int. J. Mach. Learn. Cyb. 2018, 9, 1839-1855. [CrossRef]

35. Zhang, C.; Li, D.Y.; Broumi, S.; Sangaiah, A. Medical diagnosis based on single-valued neutrosophic probabilistic rough multisets over two universes. Symmetry 2018, 10, 213. [CrossRef]

36. Abdel-Basset, M.; Manogaran, G.; Mohamed, M.; Chilamkurti, N. Three-way decisions based on neutrosophic sets and AHP-QFD framework for supplier selection problem. Future. Gener. Comp. Sy. 2018, 89, 19-30. [CrossRef]

37. Lin, J.; Yang, H.L.; Li, S.G. Three-way decision based on decision-theoretic rough sets with single-valued neutrosophic information. Int. J. Mach. Learn. Cyb. 2019, 1-9. [CrossRef]

38. Abdel-Basset, M.; Mohamed, M. The role of single valued neutrosophic sets and rough sets in smart city: Imperfect and incomplete information systems. Measurement 2018, 124, 47-55. [CrossRef]

39. Thao, N.X.; Le, H.S.; Cuong, B.C.; Ali, M.; Lan, L.H. Fuzzy Equivalence on Standard and Rough Neutrosophic Sets and Applications to Clustering Analysis. In Information Systems Design and Intelligent Applications; Advances in Intelligent Systems and Computing, AISC; Springer: Berlin, Germany, 2018; Volume 672, pp. 834-842.

40. Qian, Y.H.; Liang, J.Y.; Yao, Y.Y.; Dang, C.Y. MGRS: A multi-granulation rough set. Inform. Sci. 2010, 180, 949-970. [CrossRef]

41. Qian, Y.H.; Li, S.Y.; Liang, J.Y.; Shi, Z.Z.; Wang, F. Mgrs: Pessimistic rough set based decisions: A multigranulation fusion strategy. Inf. Sci. 2014, 264, 196-210. [CrossRef] 
42. Qian, Y.H.; Liang, X.Y.; Lin, G.P.; Guo, Q.; Liang, J.Y. Mgrs: Local multigranulation decision-theoretic rough sets. Int. J. Approx. Reason. 2017, 82, 119-137. [CrossRef]

43. Wong, S.K.M.; Ziarko, W. Comparison of the probabilistic approximate classification and the fuzzy set model. Fuzzy Set. Syst. 1987, 21, 357-362. [CrossRef]

44. Yao, Y.Y. Three-way decisions with probabilistic rough sets. Inf. Sci. 2010, 180, 341-353. [CrossRef]

45. Yao, Y.Y. The superiority of three-way decisions in probabilistic rough set models. Inf. Sci. 2011, 181, 1080-1096. [CrossRef]

46. Zhang, C.; Li, D.Y.; Mu, Y.M.; Song, D. An interval-valued hesitant fuzzy multigranulation rough set over two universes model for steam turbine fault diagnosis. Appl. Math. Model. 2017, 42, 1803-1816. [CrossRef]

47. Zhang, C.; Li, D.Y.; Zhai, Y.H.; Yang, Y.H. Multigranulation rough set model in hesitant fuzzy information systems and its application in person-job fit. Int. J. Mach. Learn. Cyb. 2019, 10, 719-729. [CrossRef]

48. Sun, B.Z.; Ma, W.M.; Li, B.J.; Li, X.N. Three-way decisions approach to multiple attribute group decision-making with linguistic information-based decision-theoretic rough fuzzy set. Int. J. Approx. Reason. 2018, 93, 424-442. [CrossRef]

49. Sun, B.Z.; Ma, W.M.; Chen, X.T.; Li, X.N. Heterogeneous multigranulation fuzzy rough set-based multiple attribute group decision-making with heterogeneous preference information. Comput. Ind. Eng. 2018, 122, 24-38. [CrossRef]

50. Zhan, J.M.; Sun, B.Z.; Alcantud, J.C.R. Covering based multigranulation (I,T)-fuzzy rough set models and applications in multi-attribute group decision-making. Inf. Sci. 2019, 476, 290-318. [CrossRef]

51. Zhang, L.; Zhan, J.M.; Xu, Z.S. Covering-based generalized IF rough sets with applications to multi-attribute decision-making. Inf. Sci. 2019, 478, 275-302. [CrossRef]

52. Liang, D.C.; Liu, D. A novel risk decision-making based on decision-theoretic rough sets under hesitant fuzzy information. IEEE. T. Fuzzy. Syst. 2015, 23, 237-247. [CrossRef]

53. Liu, D.; Liang, D.C.; Wang, C.C. A novel three-way decision model based on incomplete information system. Knowl.-Based Syst. 2016, 91, 32-45. [CrossRef]

54. Liang, D.C.; Xu, Z.S.; Liu, D.; Wu, W. Method for three-way decisions using ideal TOPSIS solutions at Pythagorean fuzzy information. Inf. Sci. 2018, 435, 282-295. [CrossRef]

55. Xu, W.H.; Guo, Y.T. Generalized multigranulation double-quantitative decision-theoretic rough set. Knowl.-Based Syst. 2016, 105, 190-205. [CrossRef]

56. Zhang, C.; Li, D.Y.; Mu, Y.M.; Song, D. A Pythagorean fuzzy multigranulation probabilistic model for mine ventilator fault diagnosis. Complexity 2018, 2018, 7125931. [CrossRef]

57. Zhang, C.; Li, D.Y.; Liang, J.Y. Multi-granularity three-way decisions with adjustable hesitant fuzzy linguistic multigranulation decision-theoretic rough sets over two universes. Inf. Sci. 2020, 507, 665-683. [CrossRef]

58. Medina, J.; Ojeda-Aciego, M. Multi-adjoint t-concept lattices. Inf. Sci. 2010, 180, 712-725. [CrossRef]

59. Takacs, A.; Kovacs, L.; Rudas, I.J.; Precup, R.E.; Haidegger, T. Models for force control in telesurgical robot systems. Acta. Polytech. Hung. 2015, 12, 95-114.

60. Gil, R.P.A.; Johanyak, Z.C.; Kovacs, T. Surrogate model based optimization of traffic lights cycles and green period ratios using microscopic simulation and fuzzy rule interpolation. Int. J. Artif. Intell. 2018, 16, $20-40$.

61. Smarandache, F.; Colhon, M.; Vladutescu, S.; Negrea, X. Word-level neutrosophic sentiment similarity. Appl. Soft. Comput. 2019, 80, 167-176. [CrossRef]

62. Kandasamy, I.; Vasantha, W.B.; Obbineni, J.M.; Smarandache, F. Sentiment analysis of tweets using refined neutrosophic sets. Comput. Ind. 2020, 115, 103180. [CrossRef]

63. Liao, J.; Wang, S.G.; Li, D.Y. Identification of fact-implied implicit sentiment based on multi-level semantic fused representation. Knowl.-Based Syst. 2019, 165, 197-207. [CrossRef]

64. Zhai, Y.H.; Li, D.Y.; Zhang, J. Variable decision knowledge representation: A logical description. J. Comput. Sci. 2018, 25, 161-169. [CrossRef]

65. Zhai, Y.H.; Li, D.Y. Knowledge structure preserving fuzzy attribute reduction in fuzzy formal context. Int. J. Approx. Reason. 2019, 115, 209-220. [CrossRef]

66. Guo, Y.T.; Tsang, E.C.C.; Xu, W.H.; Chen, D.G. Local logical disjunction double-quantitative rough sets. Inf. Sci. 2019, 500, 87-112. [CrossRef] 
67. Zhang, S.X.; Li, D.Y.; Zhai, Y.H.; Kang, X.P. A comparative study of decision implication, concept rule and granular rule. Inf. Sci. 2020, 508, 33-49. [CrossRef]

68. Zhao, C.J.; Wang, S.G.; Li, D.Y. Exploiting social and local contexts propagation for inducing Chinese microblog-specific sentiment lexicons. Comput. Speech. Lang. 2019, 55, 57-81. [CrossRef]

69. Zhao, C.J.; Wang, S.G.; Li, D.Y. Multi-source domain adaptation with joint learning for cross-domain sentiment classification. Knowl.-Based Syst. 2019, 1-16. [CrossRef]

(C) 2020 by the authors. Licensee MDPI, Basel, Switzerland. This article is an open access article distributed under the terms and conditions of the Creative Commons Attribution (CC BY) license (http:/ / creativecommons.org/licenses/by/4.0/). 